\title{
THE DISTRIBUTION AND TYPOLOGICAL CLASSIFICATION OF INCIDENCE/MORTALITY RATES AND DYNAMICS OF TUBERCULOSIS IN RUSSIA, 2006-2017
}

\author{
Svetlana Malkhazova' ${ }^{1}$ Natalia Shartova', Razia Gaida ${ }^{2,3}$, Adlai Davids ${ }^{2,3}$, Vladimir Tikunov' ${ }^{1}$, Dmitry Orlov ${ }^{\text {1* }}$ \\ 'Lomonosov Moscow State University, Moscow, Russia \\ ${ }^{2}$ Human Sciences Research Council, Port Elizabeth, South Africa \\ ${ }^{3}$ Nelson Mandela University, Port Elizabeth, South Africa \\ *Corresponding author: orlovmsu@gmail.com \\ Received: November 26 $6^{\text {th }}, 2019$ / Accepted: February 16 ${ }^{\text {th }}, 2021 /$ Published: April $1^{\text {st }}, 2021$ \\ https://DOI-10.24057/2071-9388-2020-202
}

\begin{abstract}
Despite the achieved success in the fight against tuberculosis, the disease remains an immediate problem for a number of countries including Russia. To a large extent, the reasons for the high incidence and mortality of the population are not only medical but also social in nature, which leads to the emergence of geographical patterns in the spread of the disease. The purpose of the study is to identify the spatio-temporal conditions that shape the epidemiological situation of tuberculosis in Russia at both the national and regional levels. Using GIS technologies, an analysis of the current spread of the infection in the Russian Federation was carried out based on data for the period from 2006 through 2017 . Typological classification of regions according to the dynamics and magnitude of the incidence rate has been developed. Based on the cartographic analysis that was carried out, regions with the most unfavorable tuberculosis situation in the Russian Federation were identified for a more detailed study at the municipal level.
\end{abstract}

KEY WORDS: tuberculosis, Russia, mapping, spatial analysis, typological classification, dynamics of incidence and mortality

CITATION: Svetlana Malkhazova, Natalia Shartova, Razia Gaida, Adlai Davids, Vladimir Tikunov, Dmitry Orlov (2021). The Distribution And Typological Classification Of Incidence/Mortality Rates And Dynamics Of Tuberculosis In Russia, $2006-2017$. Geography, Environment, Sustainability, Vol.14, No 1, p. 241-250 https://DOl-10.24057/2071-9388-2020-202

ACKNOWLEDGEMENTS: The reported study was funded by Russian Foundation for Basic Research (RFBR) and National Research Foundation (NFR) (South Africa) according to the research project № 19-55-60003, by RFBR according to the research project № 18-05-60037 and by NRF according to the research project №118913 with reference number RUSA180626348966. Dmitry Orlov was partly supported by Moscow State University Grant for Leading Scientific Schools «Depository of the Living Systems» within the MSU Development Program.

Conflict of interests: The authors reported no potential conflict of interest.

\section{INTRODUCTION}

Tuberculosis is one of the most pressing and challenging health issues in the world as well as a sociomedical problem for many countries. Tuberculosis affects $1 / 3$ of the world population (Shelkova, Romanenko 2013). According to the World Health Organization (WHO), 7.1 million people were diagnosed with TB for the first time in 2019 and up to 7.0 million - in 2018, which is a large increase from 6.4 million in 2017 and 5.7-5.8 million annually in the period 2009-2012 (Global tuberculosis report 2020). The infection returns to industrialized countries in a more severe and life-threatening form (Vasilieva et al. 2017). People are generally susceptible to tuberculosis, as only $1-3 \%$ of people have a genetically determined resistance to the pathogen (Shelkova, Romanenko 2013).

The goal set by the World Health Organization for the world community is to constantly reduce mortality and incidence among the population. It is planned to reduce mortality by 2025 by $75 \%$ compared to 2015 and incidence by 50\% (The WHO End TB ... 2014; Krasnov et al. 2016). In that context, a significant number of recent studies have been devoted to the diagnosis, prevention and treatment of tuberculosis including latent forms, as well as the study of the body's reactions to the pathogen (Berry et al. 2013; Alsultan, Peloquin 2014; Lee et al. 2015; Lyon, Rossman 2017; Manina et al. 2017).

Among other things, there is a severe problem of the combination of HIV infection and tuberculosis. According to $\mathrm{WHO}$, most of the countries with significant HIV/TB coinfection are located in Africa, the Republic of South Africa being one of the most affected countries (Global tuberculosis report 2020). A particular danger in this case is the difficulty of diagnosing tuberculosis in patients with HIV which, as a result, leads to a high mortality rate (Hosseinipour et al. 2016; Mbu et al. 2018). The problem of coinfection of these two diseases is also associated with the currently topical issue of drug resistance of the causative agent of tuberculosis and the need to create new drugs.

The Russian Federation is among the countries most severely affected by tuberculosis as every third patient in the WHO European Region is from Russia (Vasilieva et al. 2017). On average, more than 100 new cases of the disease appear and about 20 people die of tuberculosis daily in Russia (Nechaeva 
2018). In 2017, 70.9 thousand people with active tuberculosis were registered on the territory of the Russian Federation (the diagnosis was established for the first time in their lives) i.e. 48.3 cases per 100,000 people (Healthcare in Russia 2019). Young children are most susceptible to the disease, especially in the first months of their lives. The main source of infection for them are adults suffering from tuberculosis in active phases (Shelkova, Romanenko 2013).

Russia is a country with a high incidence of both common tuberculosis (TB) and multidrug-resistant tuberculosis (MDRTB) (Global tuberculosis report 2020). In subsequent years, the detection and control of multidrug-resistant tuberculosis will be the main focus of research both in Russia and abroad (Chung-Delago et al. 2015; Barkanova et al. 2018; Girum et al. 2018).

The importance of studying the spatial patterns of the infection spread is closely connected to the impact that the external environment has on the human body. The strategy for solving the priority tasks in the spheres of social policy and healthcare should be based on knowledge of the geography of various indicators of the epidemiological situation.

There is an obvious need to fill the existing gap in the study of spatially differentiated levels in the Russian Federation, both national and regional. Without the compilation of modern maps representing incidence and mortality in Russia as a whole and in individual regions, in particular, it is impossible to provide information support for high-quality monitoring of the epidemiological situation.

World Health Organization has assigned itself a task to ensure a wide exchange of experience in studying the level of health of the world population and introducing the best examples of health care organizations based on sciencebased preventive measures (The End TB Strategy 2014). The experience of South Africa, a country also struggling with the control and prevention of tuberculosis, will contribute to similar programmes in the Russian Federation. The methods of spatial medical-geographical analysis developed and applied in the Russian Federation can in turn inform geographically differentiated tuberculosis prevention measures in South Africa and elsewhere.

The purpose of this study is to identify the spatiotemporal features of the current epidemiological situation of tuberculosis in the Russian Federation in order to develop targeted preventive measures in the fight against the most lethal global infectious organism (World Health Organization 2020)

\section{MATERIALS AND METHODS}

The territory of Russia is divided into 85 federal subjects (hereinafter territorial units -TU): oblasts, republics, krais, autonomous oblasts, autonomous okrugs and federal cities (Fig. 1). The study used primary statistical information on incidence and mortality due to tuberculosis in the Russian Federation for the period from 2006 through 2017. Data were collected at the national level for each of the 85 regions of the Russian Federation from various sources, including publicly available statistical digests, information materials and articles by the Federal State Statistics Service (Rosstat), the Ministry of Health of the Russian Federation, Federal Research Institute for Health Organization and Informatics of Ministry of Health of the Russian Federation, The Moscow Research and Clinical Center for Tuberculosis Control of the Moscow Government Department of Health and Research Institute of Physiopulmonology at Sechenov University.

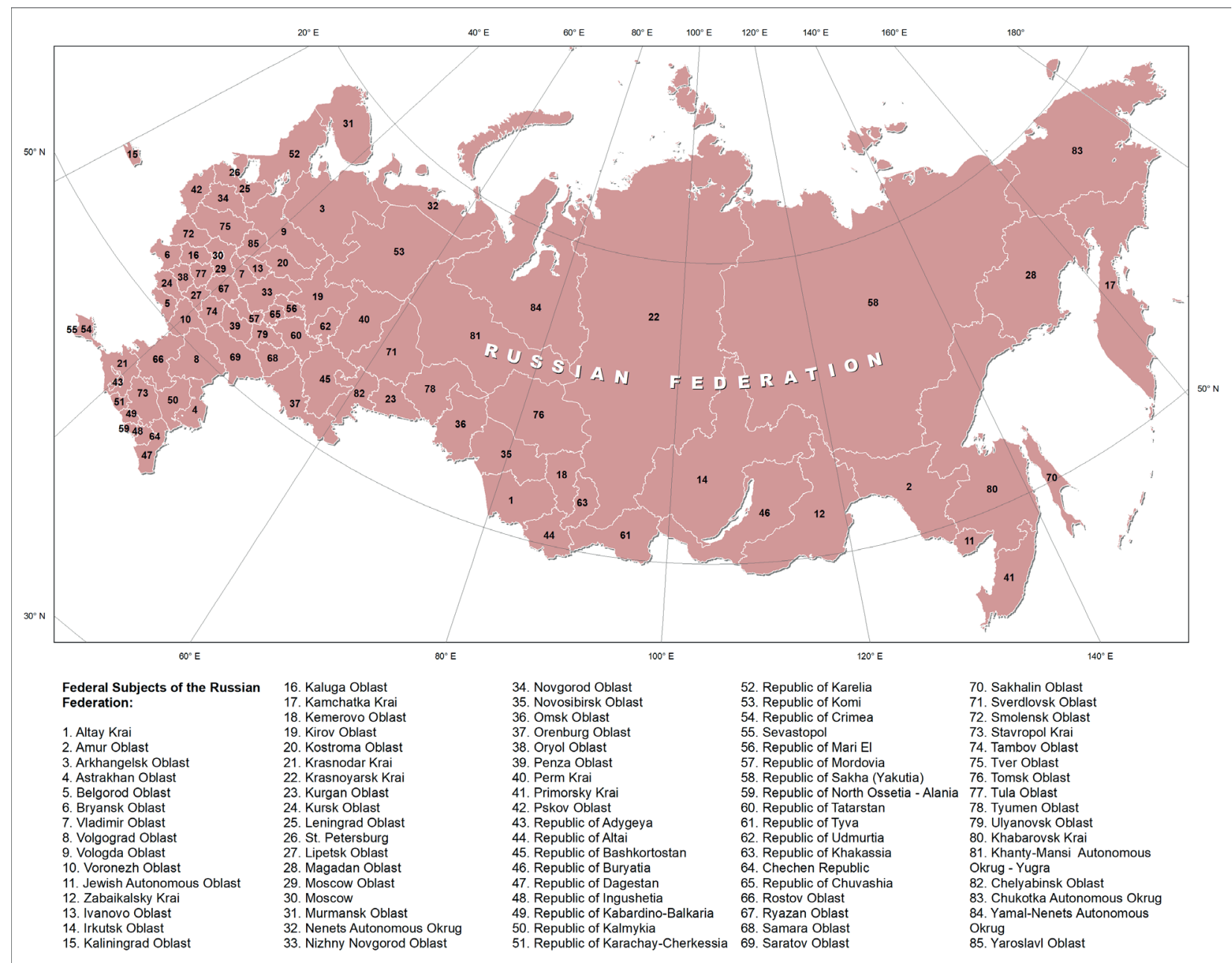

Fig. 1. Federal subjects of the Russian Federation 
Authors selected the following indicators, expressed in absolute values and per 100,000 population, that reflect the current situation of tuberculosis in Russia to the fullest extent: the incidence of all forms of tuberculosis, incidence of pulmonary tuberculosis, incidence of tuberculosis in children and mortality from tuberculosis and other consequences.

The calculation of standardized mortality rates (Everitt, Skrondal 2010) was carried out according to a direct standardization method based on the information on the absolute number of deaths from tuberculosis in the regions of the Russian Federation. This was implemented to eliminate the influence of the age and sex structure of the population. All the collected statistical materials were combined into one database in the form of attribute tables in the QGIS software. The resulting database became the basis for creating GIS layers (QGIS), which were then used to produce a series of analytical and typological maps for the period from 2006 to 2017.

This was followed by a more detailed dynamic typological classification for all of the regions of the Russian Federation for each of the analyzed indicators, which was made using the original classification algorithm with several sequential operations: normalization, weighting and dimensionality reduction for the data, all carried out by variances and mathematical expectations (Tikunov 1997). This made it possible to clarify the spatio-temporal pattern of the distribution of the incidence and mortality rates. The principal component analysis was applied to correct the original space of features distorted by cross-correlations as well as to identify latent variables that reflect the essence of a process or phenomenon.

In the applied typological algorithm, the entire set of parameters for any territorial unit (TU) is denoted by $\mathrm{X}=\{\mathrm{x} 1, \ldots, \mathrm{xN}\}$, where $\mathrm{x}-\mathrm{i}$-th $\mathrm{TU}, \mathrm{N}$ - the number of TUs. The initial TUs are represented in the form of a TU-sign matrix, which reflects the measurement of signs on TU and contains rows and columns:

$$
X=\left(\frac{x_{1}}{x_{N}}\right)=\left(x^{(1)}, \ldots, x^{(M)}\right)=\left(\begin{array}{lllll}
x_{1}^{(1)} & \cdots & x_{1}^{(j)} & \cdots & x_{1}^{(M)} \\
\cdots & \cdots & \cdots & \cdots & \cdots \\
x_{N-1}^{(1)} & \cdots & x_{N-1}^{(j)} & \cdots & x_{N-1}^{(M)} \\
x_{N}^{(1)} & \cdots & x_{N}^{(j)} & \cdots & x_{N}^{(M)}
\end{array}\right)
$$

where

$x i=\left(x_{i}^{(1)}, \ldots, x_{i}^{(M)}\right)-i$-th TU in $M$ - dimensional space of parameters

$x^{(j)}$ - j -th parameter, $x^{(j)}=\left(x_{1}^{(j)}, \ldots, x_{N}^{(j)}\right)$,

$x_{i}^{(j)}$ - the value of $j$-th parameter of $i$-th $T U$,

$i \in\{1, \ldots, N\}, j \in\{1, \ldots, M\}$.

The next stage of the TUs' classification is their pretreatment, which includes normalization, weighing and dimensionality reduction. The normalization was carried out according to the variance and mathematical expectation. The purpose of this normalization is to bring each indicator to a standard form (as a result, the mathematical expectation of any indicator becomes equal to zero, and the variance to unity). Assuming $\bar{x}^{(j)}=\frac{1}{N} \sum_{i=1}^{N} x_{j}^{(j)}$ is the estimate of mathematical expectation and $\operatorname{var}\left(x^{(j)}\right)=\frac{1}{N} \sum_{i=1}^{N}\left(x_{j}^{(j)}-\bar{x}^{-(j)}\right)$ is

the estimate of variance of $j$-th parameter, the normalization means the following recalculation:

$x_{i}^{(j)}=\frac{x_{i}^{(j)}-x^{-(j)}}{\sqrt{\operatorname{var} x^{(j)}}} \forall j \in\{1, \ldots, M\}, i \in\{1, \ldots, N\}$, T.e $\Delta_{1}=x^{-(j)}, \Delta_{2}=\sqrt{\operatorname{var}\left(x^{(j)}\right)}$

The next step includes the application of the principal component method. This method solves the problem of analyzing the existing system of attributes that describe TU. The method of principal components is used to correct the original feature space distorted by mutual correlations, reduce the amount of stored data without losing a significant part of information about TU, visualize TU in the feature space (which is achieved, for example, by displaying TU in the form of points on a plane) and reveal hidden indicators that reflect the essence of the process or phenomenon.

The simplest interpretation of the principal component method is geometric. In a multidimensional parameters space, TU are considered as points, whose cloud's geometrical arrangement, in the case of the normal distribution, resembles an M-dimensional ellipsoid. The main axes of the imaginary ellipsoid are treated as the new parameters, sorted in the descending order of the TUs dispersions along the axes.

The most general relation is used to calculate the distance for M quantitative parameters:

$$
d_{e}\left(x_{i}, x_{j}\right)=\sqrt{\sum_{x=1}^{M}\left(x_{i}^{(x)}-x_{j}^{(x)}\right)^{2}}
$$

Our classifications aimed to obtain homogeneous TU groups in M-dimensional attribute space, i.e., possible "types" of TU. For this, the largest distance is selected from the obtained Euclidean distances and the two territorial units that it connects become the nuclei of the homogeneous clusters. Clusters are formed by the distribution of the remaining territories between the two cores according to the minimum Euclidean distances. In the case of a larger number of clusters, to determine the third and all subsequent cores, each of all remaining territorial units is substituted in the form of a core, and the rest is distributed between the three cores according to the minimum distance. The procedure for determining the 4-th core and formation of a typology with 4 types is similar to that described above. The maximum possible number of groups $t_{\max }$ is specified in advance, similarly to the minimum value $t$. The resulting number of groups can be analyzed using the absolute and relative heterogeneity coefficients and, thus, the optimal number of clusters can be chosen:

$$
\begin{aligned}
& A_{k}=\frac{100\left\{\sum_{k=1}^{K} \sum_{j=1}^{n} \sum_{i=1}^{n}\left[\sum_{p=1}^{p}\left(x_{i p}-x_{j p}\right)^{2}\right]^{1 / 2} I_{i k} I_{j k}\right\}}{\sum_{i=1}^{t_{\max }}\left[\sum_{p=1}^{P}\left(x_{i p}-x_{j p}\right)^{2}\right]^{1 / 2}}, \\
& k=t_{\min }, t_{\min }+1, \ldots, t_{\max } ; \\
& O_{k}=\frac{100\left\{\sum_{k=1}^{K} \sum_{j=1}^{n} \sum_{i=1}^{n}\left[\sum_{p=1}^{p}\left(x_{i p}-x_{j p}\right)^{2}\right]^{1 / 2} I_{i k} I_{j k}\right\}}{\sum_{i=1}^{t_{\max }} \sum_{j=1}^{n} \sum_{i=1}^{n}\left[\sum_{p=1}^{P}\left(x_{i p}-x_{j p}\right)^{2}\right]^{1 / 2} I_{i k} I_{j k}}, \\
& k=t_{\min }, t_{\min }+1, \ldots, t_{\max }-1 .
\end{aligned}
$$

where $\mathrm{k}$ - the number of identified groups, $\mathrm{p}$ - the number of the orthogonalized coefficients to calculate distances, $t_{\text {min' }} t_{\text {max }}$ - the maximal and the minimal number of groups, $l_{i k^{-}}$indicator (binary), pointing to the presence (1) or absence (0) of TUi in group $k$.

A sharp increase in $A_{k}$ or $O_{k}$ with a decrease in the number of the identifiable clusters indicates an increase in heterogeneity within the identified clusters, while, on the contrary, a smooth increase in the coefficients is a sign of its uniform increase. The threshold followed by a sharp increase in heterogeneity can be optimally taken as the final number of clusters. In our analysis, the isolation of five clusters in all calculations turned out to be optimal (Tikunov 1997).

The «TB hot spots» of the Russian Federation were determined using cluster analysis. Several groups of regions 
with similar mortality and incidence rates among the adult population were identified. The k-means grouping was used as a clustering method. As a result of the iterations of the cluster analysis, it was recognized that it was optimal to single out three groups characterized by a gradual increase in both the incidence and mortality rates among the entire population, which reflects the unfavourability of the epidemic situation.

\section{RESULTS AND DISCUSSION}

Incidence and mortality from tuberculosis and its temporal dynamics in the Russian Federation

Tuberculosis in Russia remains one of the country's main epidemiological problems. At the same time, during the analyzed period, there was a tendency towards a decrease in incidence and mortality from tuberculosis for all considered indicators. The incidence rate was 82.6 per 100,000 population in 2006 but over 10 years it has dropped by almost half to 48.3 per 100,000 population in 2017.

The mortality is also characterized by a rapid decline, from 20 per 100,000 population in 2006 to 6.5 per 100,000 population in 2017. While there is a decrease in mortality from TB, the «all-cause fatality rate of TB» indicator is rising. The fatality rate depends on a number of factors (for example, the clinical form of tuberculosis, patient's age, social status and presence of coexisting pathologies) and thus indirectly reflects the effectiveness of treatment of patients with tuberculosis.

The decrease in the incidence rate among children was less pronounced. It remained at 16.0 per 100,000 population from 2006 through 2012; since 2013 a decrease in the number of cases was constantly observed and the incidence rate in 2017 was registered at 9.7 .

It should be noted that the decrease in the incidence of tuberculosis occurred following a wider roll-out of tests for latent TB infection and a decrease both in the number of postmortem diagnostics and one-year mortality. According to the Central Research Institute for Organization and Informatization of Healthcare of the Ministry of Health of the Russian Federation, the periodic screenings for tuberculosis cover about $70 \%$ of the population. The best timely detection of tuberculosis is observed in Volga, Ural, Siberian Far Eastern regions and to a lesser extent
Central and Southern federal districts. The low detection rate of tuberculosis corresponds to the Northwest region. The quality of detection rate in the North Caucasian Federal District requires clarification.

\section{Spatial heterogeneity of tuberculosis}

Considering the territorial heterogeneity in the distribution of the long-term average annual incidence rate of tuberculosis (all forms) for the period from 2006 through 2017, it should be noted that there is a pronounced differentiation between the European part of the Russian Federation and the rest of the country (Fig. 2). The incidence can be defined as «significantly below average» or «below average» in almost all regions of the European part of the Russian Federation except for Kaliningrad Oblast and the border regions of Russia (Pskov, Smolensk and Bryansk oblasts). The most burdened regions (where the incidence rate is «significantly above average») are located beyond the Urals, mainly in the southern border regions of the Russian Federation (Kurgan, Tyumen, Omsk oblasts and other regions). This category also includes almost all of the regions of Eastern Siberia and the Far East except for the Republic of Sakha (Yakutia), Magadan and Sakhalin oblasts, and Kamchatka Krai.

While analyzing the structure of cases, according to the Central Research Institute for Organization and Informatization of Health Care, it should be noted that among the newly registered patients with tuberculosis, $86.4 \%$ belong to the permanent residents of Russia. The proportion of foreign nationals from the countries of the former USSR is about 3.5\%; the highest proportion of foreign citizens with tuberculosis is observed in Moscow (31.7\%) and St. Petersburg (14.5\%). The share of tuberculosis cases in the institutions of the Federal Penitentiary Service (FSIN) accounts for $8 \%$.

Among the affected people, about $65 \%$ are men (excluding the cases in the institutions of the Federal Penitentiary Service, where there are mainly young men), and about 35\% are women. The age structure is as follows: $30 \%$ of cases are among the 18-34 age group, about 30\% are among the 35-44 age group and 35\% correspond to the group of 45 years old and more.

While there are positive trends in the situation with tuberculosis in the Russian Federation as a whole, the problem of multidrug-resistant tuberculosis (MDR-TB) remains relevant as the number of MDR-TB patients is growing. The proportion

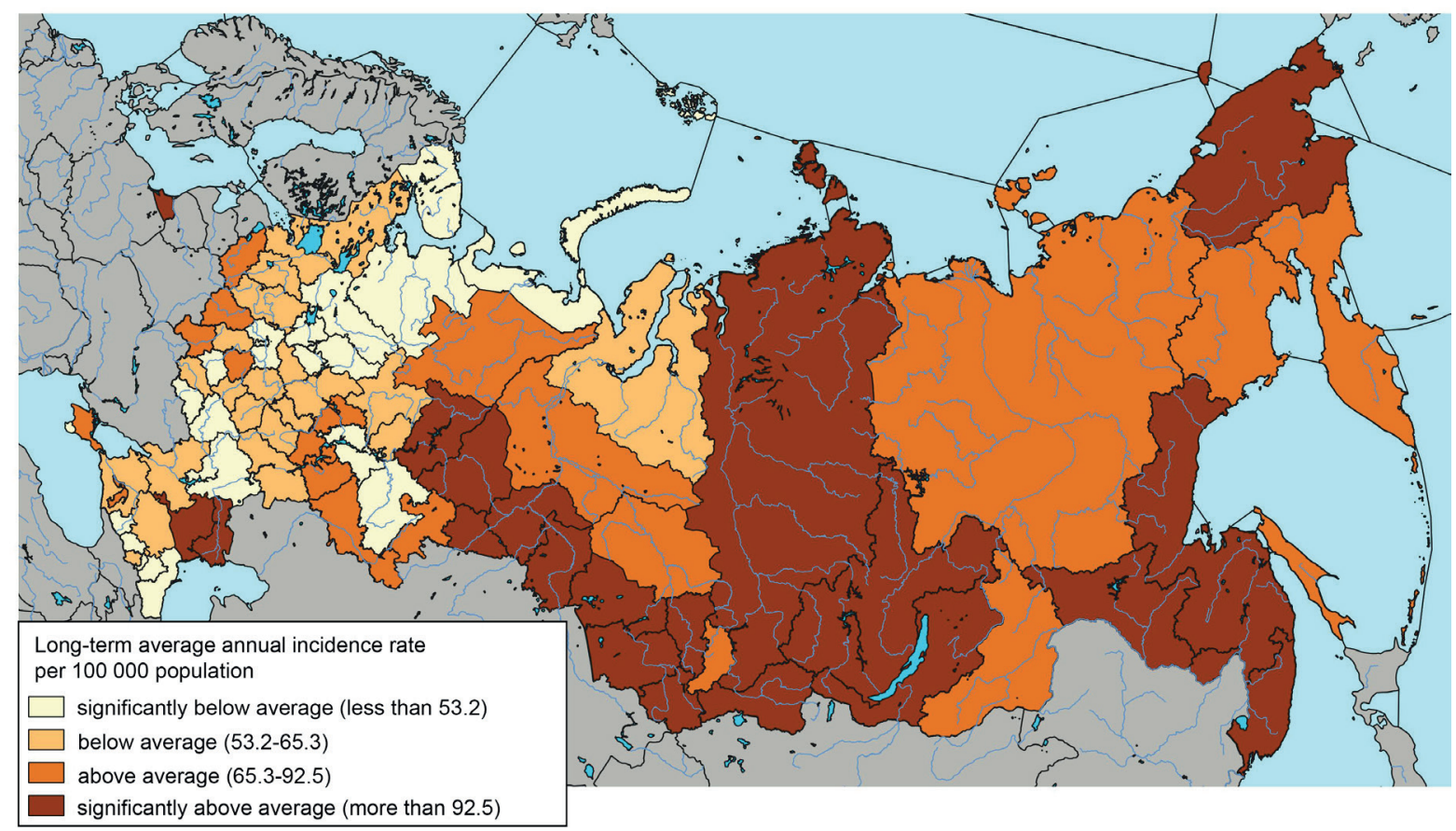

Fig. 2. Long-term average annual incidence rate of TB (all forms) for the period from 2006 to 2017 
of patients with MDR-TB among patients with pulmonary tuberculosis was 30\% in 2010 and 54\% in 2017. Among newly diagnosed TB patients, the proportion of patients with MDR-TB was 14\% and 27\% in 2010 and 2017, respectively. Among those who were registered and died of tuberculosis in 2017, 44\% of patients had MDR-TB; among those who died from other causes, 27.5\% had MDR-TB.

The modern paradigm of epidemiological research provides for a joint analysis of the spread of tuberculosis and HIV infection. This is because there has been a drastic increase in the number of tuberculosis cases in patients previously infected with HIV over the past decades.

While there is a stable decrease in the incidence of tuberculosis, at the same time an increase in the incidence of HIV is taking place (Fig. 3). The turning point came in 2014 when the number of people with identified HIV antibodies exceeded the number of cases of tuberculosis (per 100,000 population) (Central Research Institute for Health Organization and Informatization of the Ministry of Health of the Russian Federation 2020). A similar situation in terms of mortality occurred a year later, in 2015. Thus, there is still a risk of additional cases of tuberculosis among HIVinfected people due to a decline in their immune protection.
The spread of HIV/TB coinfection is observed primarily in the age groups of 25-44 years, which is typical for all regions of the Russian Federation. Moreover, every second patient registered as having died of HIV infection dies from the progression of tuberculosis at the late stages of HIV infection (Central Research Institute for Health Organization and Informatization of the Ministry of Health of the Russian Federation 2020).

The incidence rate among children by regions overall repeats the picture typical for the entire population of Russia (Fig. 4). The European Russia can be characterized as the most favorable territory (except for Kaliningrad, Bryansk, Yaroslavl oblasts as well as the Republics of Kalmykia and Adygea). A relatively positive situation is observed in the regions of Western Siberia. At the same time, the incidence rate is above average or significantly above average throughout Eastern Siberia and the Far East (except for Sakhalin and Amur oblasts, Khabarovsk and Zabaikalsky Krais).

Considering the age structure of tuberculosis incidence in children of 0-14 years old, it should be noted that approximately $40-50 \%$ of cases appear to be in the age group of 7-10 years, about $40 \%$ at the age of 3-6 years and about $10 \%$ at the age of 1-2 years.

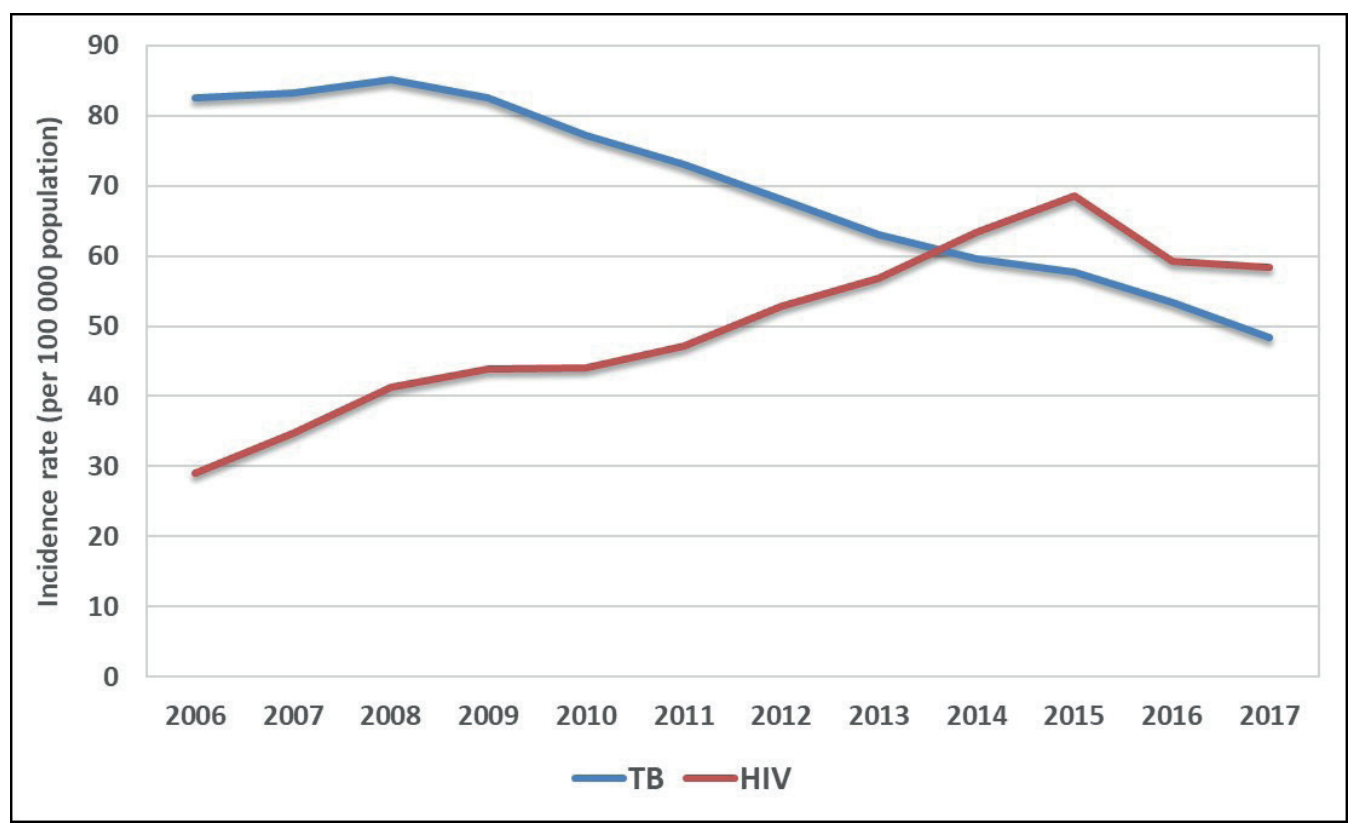

Fig. 3. TB and HIV incidence rate (per 100000 population) in Russia for the period from 2006 to 2017

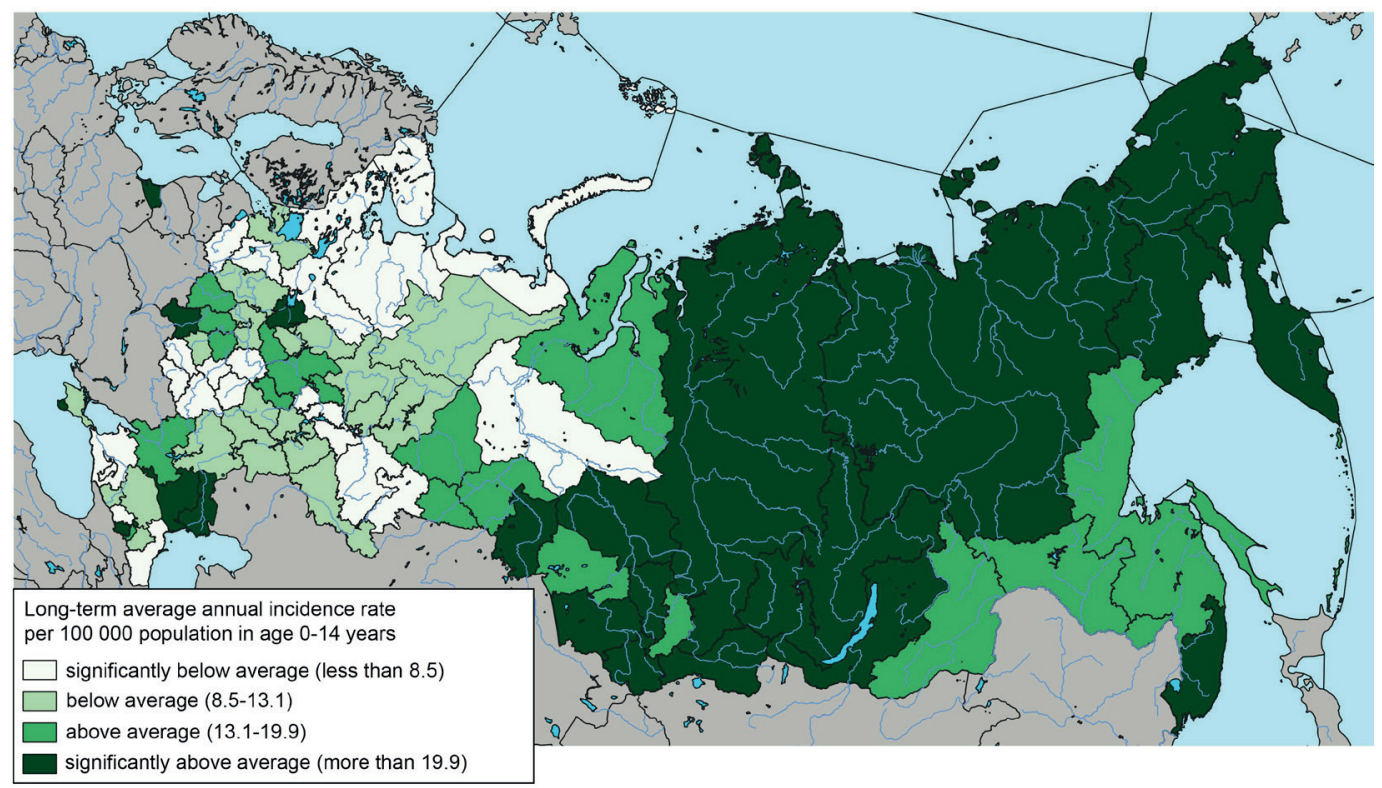

Fig. 4. Long-term average annual incidence rate of TB among children 0-14 years for the period from 2006 to 2017 
Although the spatial pattern of mortality from tuberculosis in general repeats the distribution of the incidence, it is far more mosaic and uneven (Fig. 5).

A number of regions that have both high incidence and high mortality rates stand out, for example, Krasnoyarsk and Altai Krais, Omsk and Tyumen oblasts, and some others. The opposite situation is observed in other regions where high incidence correspond to low mortality rates, for example, the Republic of Sakha (Yakutia), Magadan and Tomsk oblasts. The peak of mortality from tuberculosis in the regions of the Russian Federation falls at the age of 35-64 years.

In general, it is possible to conclude based on all the considered indicators, that the unfavorable situation in terms of tuberculosis is confined to the Asian part of Russia.

Typological classification of regions of the Russian Federation according to the level and dynamics of the epidemic indicators of tuberculosis

A typological classification based on dynamic characteristics made it possible to identify regional patterns in the epidemiological situation over the considered time interval as well as some features, representative of the territory of the Russian Federation as a whole.

Analysis of the created typological map on the incidence of tuberculosis in the entire population for the period from 2006 to 2017 showed that all identified types are characterized by a tendency towards a decrease in incidence (Fig. 6). The greatest changes can be detected in the regions, belonging to the following types: type II (high incidence rate accompanied by a pronounced downward trend) represented by the Republic of Tyva, Primorsky Krai, Jewish Autonomous Oblast; type III (decrease in the incidence rate since 2007) represented by only 14 regions located mainly beyond the Urals; and type IV (average incidence rate) represented by 11 regions mainly located along the southern border of the Russian Federation and Kaliningrad region. Types $V$ and $V I$ have an average incidence rate with a gradual downward trend. At the same time, the incidence rate for regions included in type $V$ (mainly the regions of the European Russia and Yamalo-Nenets Autonomous Okrug) is slightly higher than for the regions included in type VI. Type I is characterized by the lowest incidence rate with a similar decreasing trend and includes 18 European regions of the country.
In general, the correspondence of the spatial pattern of the long-term average annual incidence rate (obtained as a result of a cartographic analysis) to the selected typological units taking into account the observed dynamics of the indicator, suggests that the epidemiological situation is reflected objectively and that both methodological techniques were used appropriately.

Considering the typological classification for the incidence rate of pulmonary tuberculosis, the observed spatio-temporal pattern is similar to the incidence rate for all forms of TB with the difference that the selected types are characterized by a smoother decrease and the absence of any peaks (Fig. 7).

The groups identified based on the incidence of tuberculosis in children differ in terms of their dynamics (Fig. 8). Type II (high incidence rate with a zigzag downward trend) links together regions with a severely unfavorable epidemiological situation for tuberculosis in children, particularly Kamchatka Krai and Magadan Oblast. Type VI (high incidence rate with a mild downward trend) also corresponds to a number of disadvantaged regions. This type includes 8 regions located in different parts of the country (Kaliningrad Oblast, Republic of Kalmykia, Astrakhan Oblast, Republics of Altai and Tyva, Kemerovo Oblast, Primorsky Krai and Chukotka Autonomous Okrug). Type III is marked by an average incidence rate with a single spike in 2013. It includes 12 regions, almost all of which are in Siberia and the Far East except for Yaroslavl and Bryansk oblasts (which are located in European Russia). Types IV and V combine regions with an average incidence rate and a mildly pronounced downward trend. These are mostly the European regions of the Russian Federation. Type I characterizes regions with the most favorable situation demonstrating consistently low incidence rates during the entire study period. This type includes 21 regions located exclusively in European Russia

It should be noted, that the use of a typological classification for the incidence in children demonstrates a more uneven spatial pattern in comparison with the longterm average annual indicator, especially in Siberia and the Far East regions. This could be a result of fairly significant annual fluctuations of incidence in these regions.

The results of the typological classification reveal an even greater differentiation of regions in terms of mortality (Fig. 9). The most unfavorable situation was registered in only one region, i.e., the Republic of Tyva (type II, characterized by high

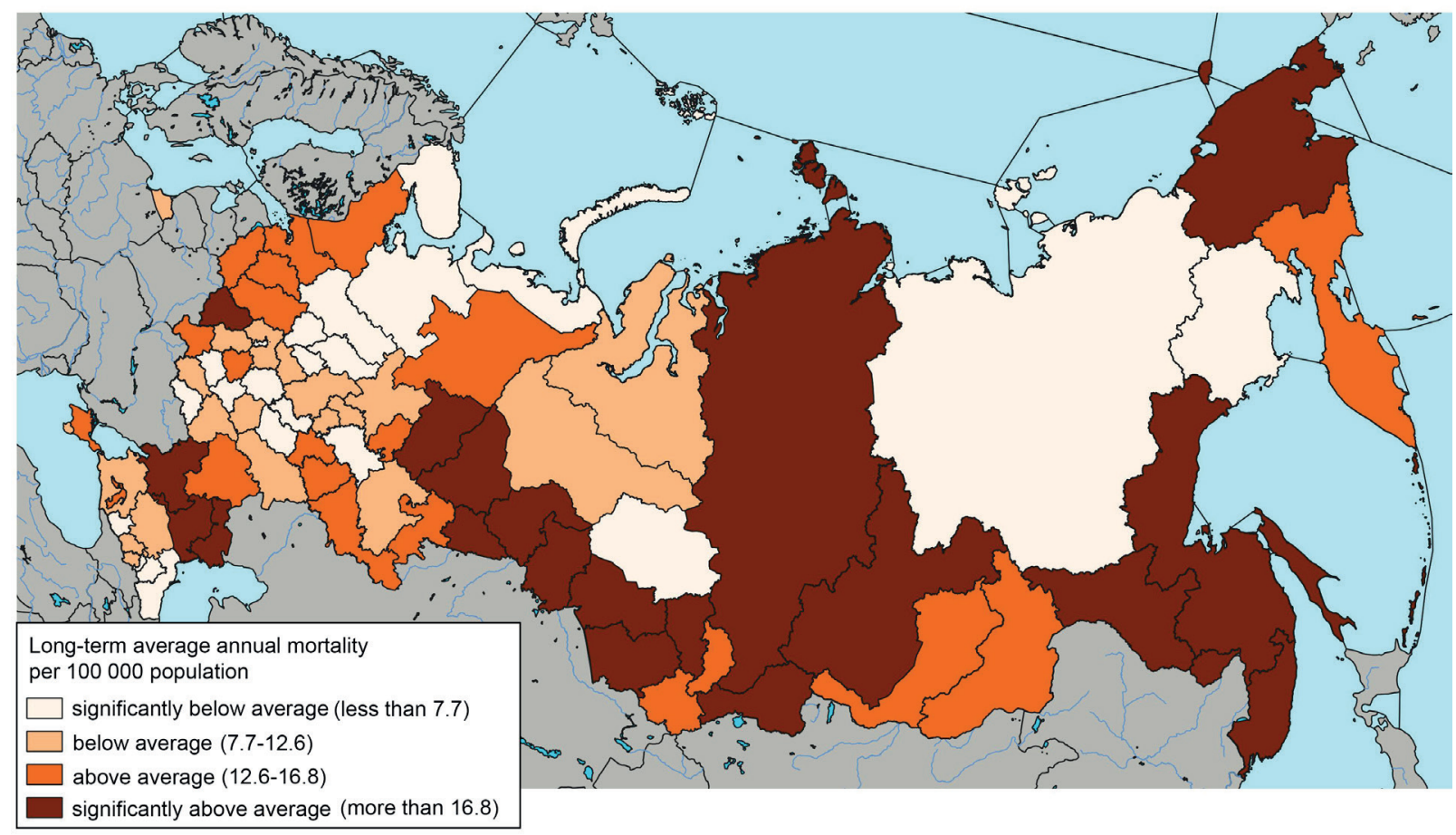

Fig. 5. Long-term average annual mortality from TB for the period from 2006 to 2017 


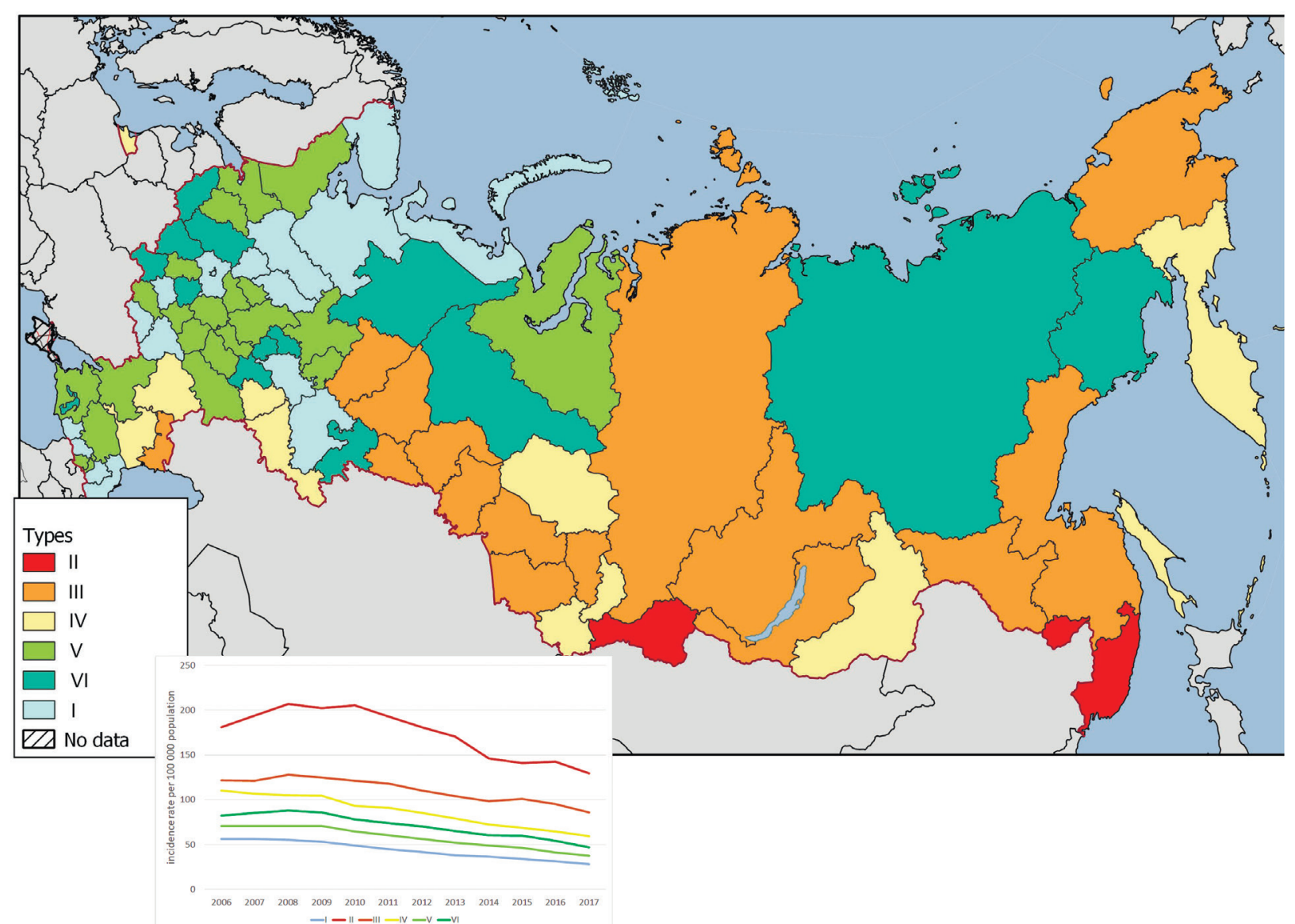

Fig. 6. Types of regions of the Russian Federation by the rate and dynamics of the incidence of TB

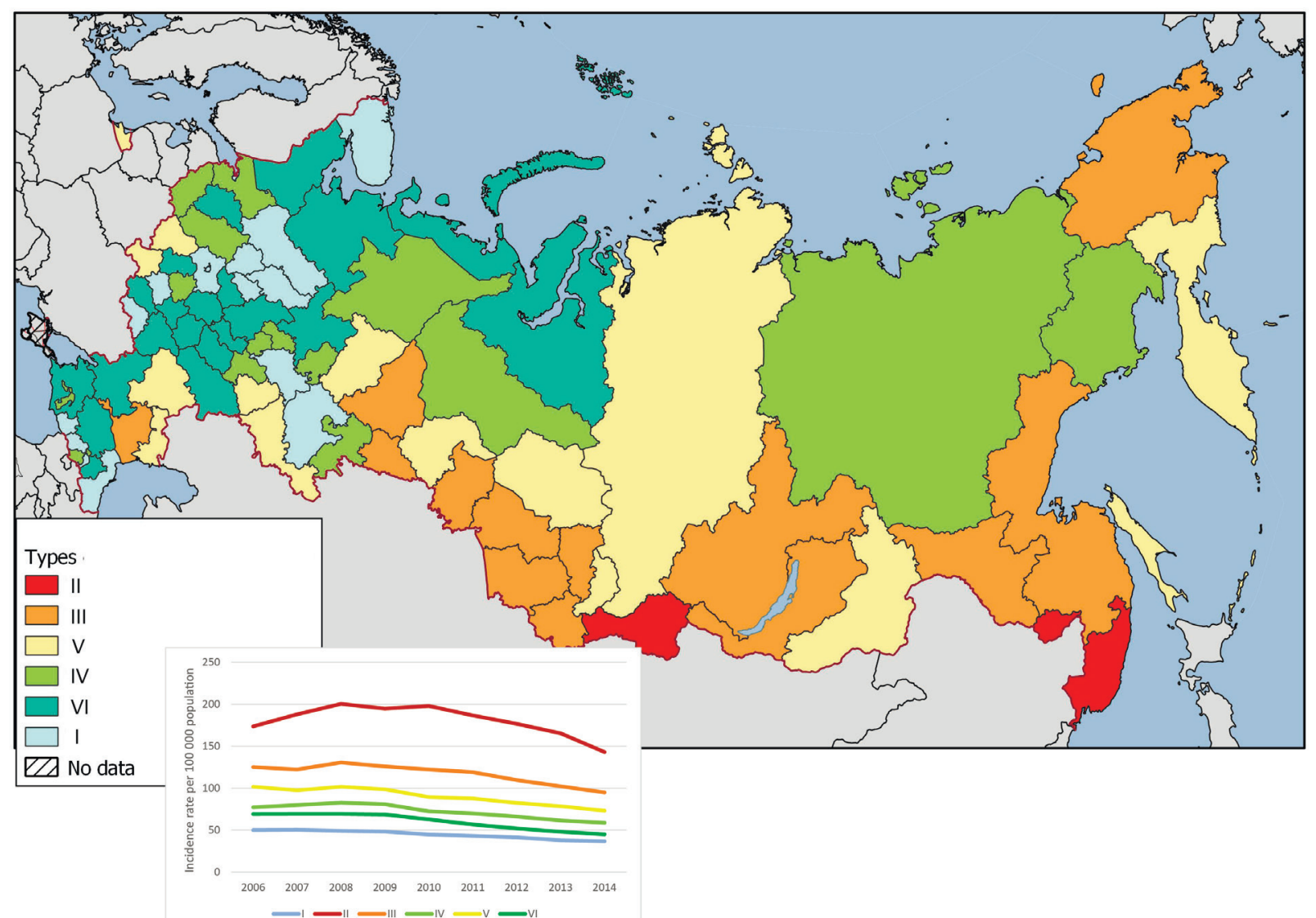

Fig. 7. Types of regions of the Russian Federation by the rate and dynamics of the incidence of pulmonary TB 


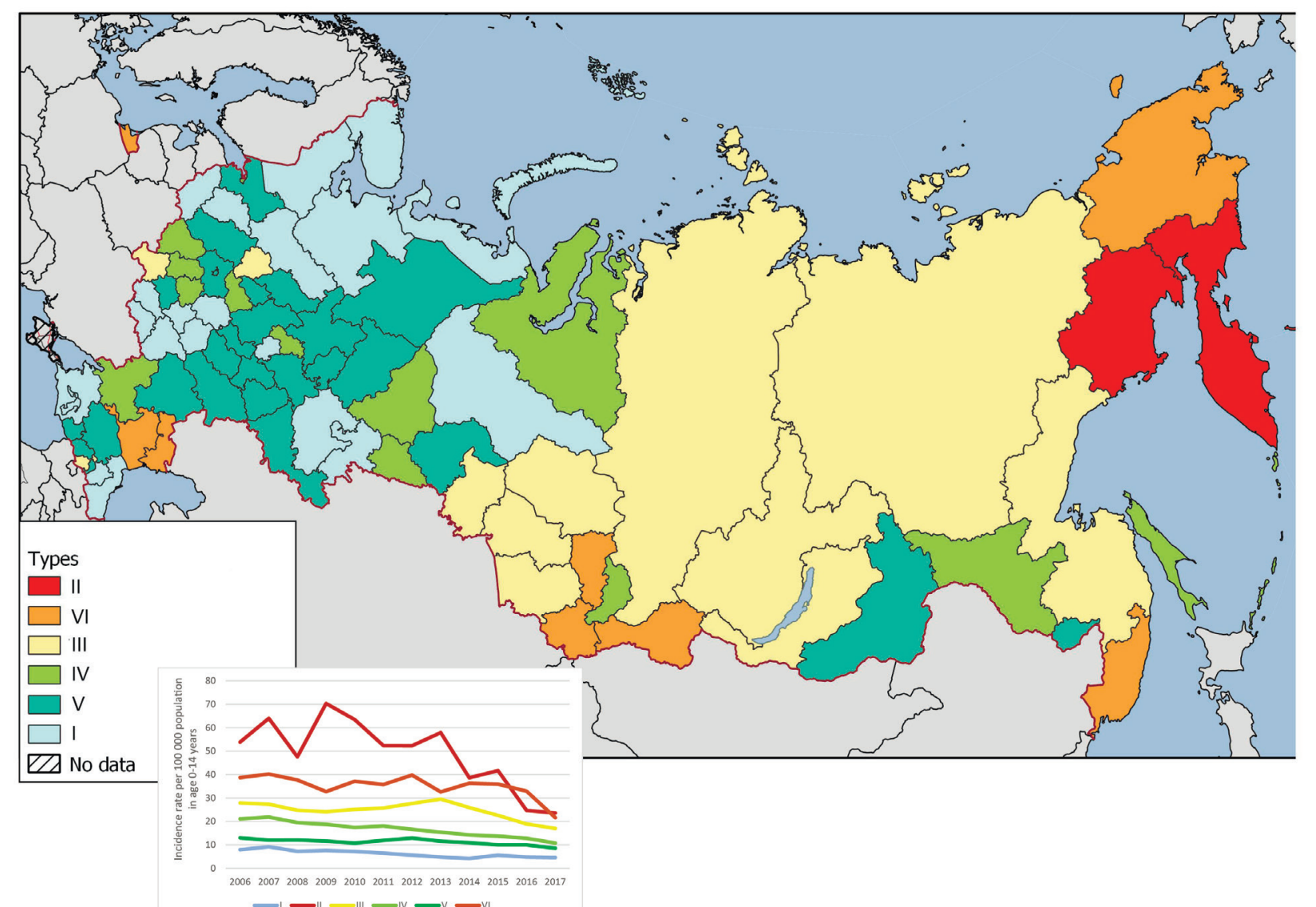

Fig. 8. Types of regions by the rate and dynamics of the incidence of TB among children 0-14 years

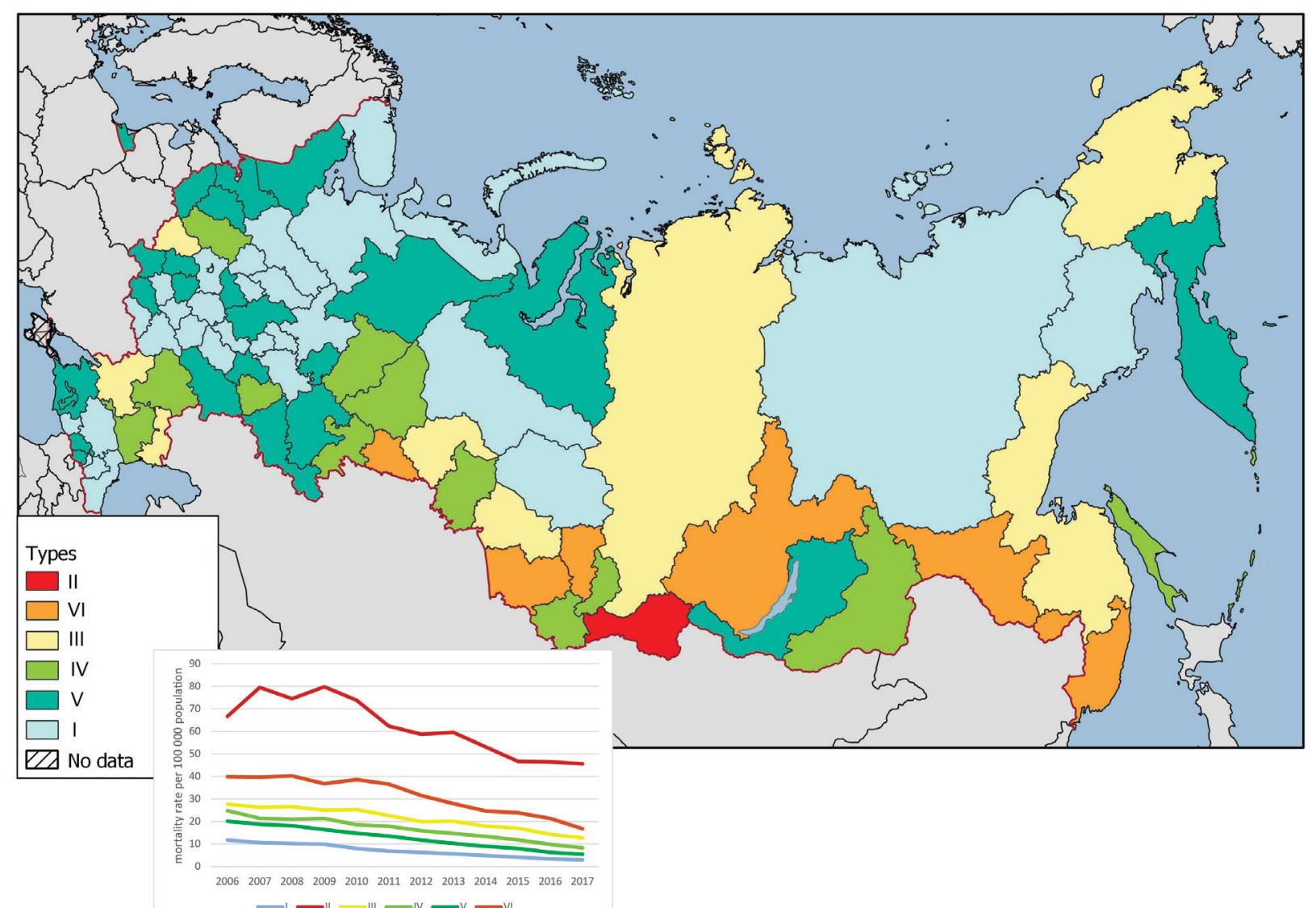

Fig. 9. Types of regions by the rate and dynamics of TB mortality 


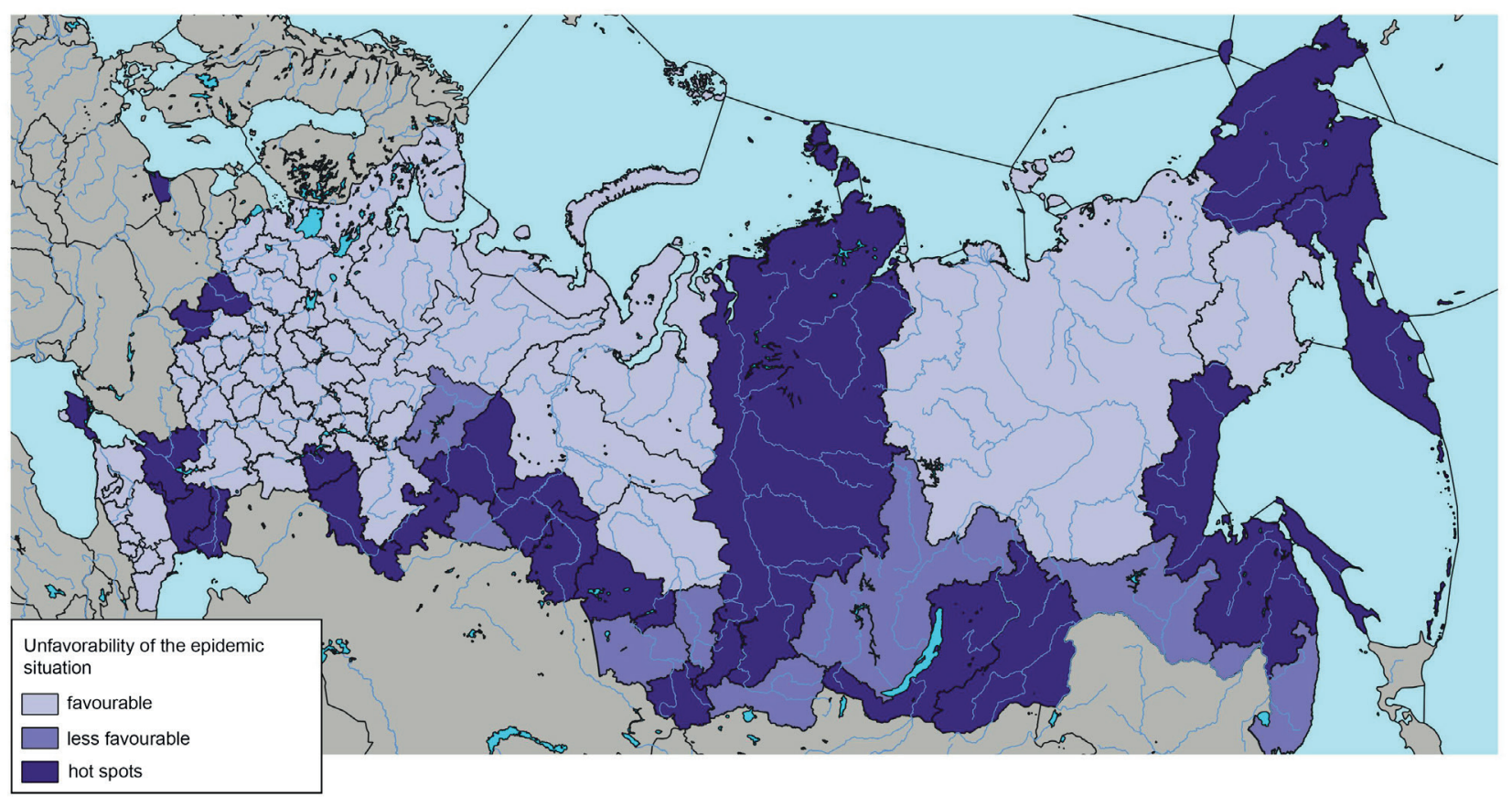

Fig. 10. The unfavourability of the epidemic situation in regions of the Russian Federation based on cluster analysis of the incidence and mortality rates (all forms of TB)

mortality with a pronounced downward trend). Type $\mathrm{VI}$ is also marked by a fairly high mortality rate but a less pronounced downward trend. This type includes 7 regions located mainly in the south of Siberia. Types III, IV, V and I differ only in magnitude and have similar tendencies towards a reduction of mortality rates. The vast majority of regions (31) belong to type I which has the lowest mortality rate.

The results of the typological classification show that the identified types differ mostly by the incidence and mortality rates but the dynamics of indicators for all types is characterized by a downward trend.

\section{Hot spot regions of the Russian Federation}

Analysis of the cartographic material created using cluster analysis (Fig. 10) made it possible to identify regions with various levels of incidence and mortality from tuberculosis over the analyzed period, i.e., the hot spot regions of Russia and the more favorable regions. The tuberculosis hot spots are found in some regions of European Russia (Kaliningrad, Smolensk, Bryansk oblasts), southern regions (Rostov Oblast, the republics of Kalmykia and Adygea), the Siberian and the Far East regions (Krasnoyarsk Krai, Novosibirsk and Omsk oblasts, Republics of Altai and Buryatia, Zabaikalsky, Khabarovsk and Kamchatka krais, Sakhalin and Magadan oblasts). Less unfavorable, average situation was revealed mainly in the regions of southern Siberia and the Far East (Primorsky Krai, Amur Oblast, Jewish Autonomous Oblast, Irkutsk Oblast,
Republic of Tyva and Altai Krai). The rest of the regions of the Russian Federation have a low degree of epidemic tension thus being the safest. The analysis made it possible to differentiate the territory of the Russian Federation and identify epidemically unsafe regions in which more detailed research is required at the municipal level. The obtained results confirm the conclusions of the cartographic analysis presented above.

\section{CONCLUSIONS}

An analysis of the current spread of tuberculosis in the Russian Federation was carried out using GIS technologies and epidemic data for the period from 2006 through 2017. As a result of the study, the spatial and temporal features of the epidemic situation in Russia were revealed in terms of the incidence of tuberculosis for the entire population (of all forms of TB), incidence of pulmonary TB, incidence of TB in children and mortality from TB. Typological classification of regions was developed based on the dynamics and magnitude of the incidence rate; it was revealed that there is an overall downward trend in incidence and mortality from TB in recent years. The highest burden regions of the Russian Federation in terms of tuberculosis with consistently high levels of incidence and mortality throughout the analyzed period were identified. The obtained results can be used for a further detailed study of the tuberculosis spread at the municipal level as well as for planning anti-tuberculosis measures in Russia.

\section{REFERENCES}

Alsultan A., Peloquin C.A. (2014). Therapeutic Drug Monitoring in the Treatment of Tuberculosis: An Update. Drugs 74, 839-854, DOI: 10.1007/s40265-014-0222-8.

Barkanova O.N., Gagarina S.G., Kaluzhenina A.A., Popkova N.L. (2018). Modern drug-resistant pulmonary tuberculosis. Bulletin of the Volgograd State Medical University, 1(65), 23-25.

Berry M., Blankley S., Graham C., Bloom C., O'Garra A. Systems approaches to studying the immune response in tuberculosis. Current Opinion in Immunology, 25(5), October 2013, 579-587, DOI: 10.1016/j.coi.2013.08.003.

Central Research Institute for Health Organization and Informatization of the Ministry of Health of the Russian Federation.https:// mednet.ru/informatizatsiya/tsentr-monitoringa-tuberkuleza\#h11-analiticheskie-obzory) [Accessed 15.10.2020].

Chung-Delgado K, Guillen-Bravo S, RevillaMontag A, Bernabe-Ortiz A (2015). Mortality among MDR-TB Cases: Comparison with DrugSusceptible Tuberculosis and Associated Factors. PLoS ONE 10(3): e0119332, DOI: 10.1371/journal.pone.0119332. 
Everitt B., Skrondal A. (2010). «Standardized mortality rate (SMR)». The Cambridge dictionary of statistics. New York: Cambridge University Press, 409.

Girum T., Muktar E., Lentiro K., Wondiye H., Shewangizaw M. (2018). Epidemiology of multidrug-resistant tuberculosis (MDR-TB) in Ethiopia: a systematic review and meta-analysis of the prevalence, determinants and treatment outcome. Trop Dis Travel Med Vaccines, 4(5), Published online 2018 Jun 14, DOI: 10.1186/s40794-018-0065-5.

Global tuberculosis report (2020). WHO, 208.

Healthcare in Russia (2019). Statistical collection / Rosstat, Moscow, 2019, 170.

Hosseinipour M.C. et al. (2016). Empiric Tuberculosis Therapy versus Isoniazid in Advanced HIV-infected Adult Outpatients Initiating Antiretroviral Therapy: a Multi-Country Randomized Controlled Trial.Lancet, 387(10024): 1198-1209, DOI: 10.1016/S0140-6736(16)00546-8.

Krasnov V.A., Revyakina O.V., Filimonov P.N., Stepanov D.V. (2016). Tuberculosis: general patterns of the epidemic process in Russia and beyond the Urals. Tuberculosis and lung diseases, 94(10): 5-11, DOI: 10.21292/2075-1230-2016-94-10-5-11.

Lee J.Y. (2015). Diagnosis and Treatment of Extrapulmonary Tuberculosis. TubercRespir Dis (Seoul), 78(2): 47-55, DOI: 10.4046/ $\operatorname{trd}$.2015.78.2.47.

Lyon S., Rossman M. (2017). Pulmonary Tuberculosis, p 285-298. In Schlossberg D (ed), Tuberculosis and Nontuberculous Mycobacterial Infections, Seventh Edition. ASM Press, Washington, DC, DOI: 10.1128/microbiolspec.TNMI7-0032-2016.

Manina V.V., Starshinova A.A., Panteleev A.M. (2017). Tuberculosis and HIV infection: the epidemic situation in Russia and the world over the past ten years, the specifics of detection and diagnosis. HIV infection and immunosuppression, 9(4): 7-16, DOI: 10.22328/2077-98282017-9-4-7-16.

Mbu E.T., Sauter F., Zoufaly A., BronsvoortBMdC, Morgan K.L., Noeske J. et al. (2018). Tuberculosis in people newly diagnosed with HIV at a large HIV care and treatment center in Northwest Cameroon: Burden, comparative screening and diagnostic yields, and patient outcomes. PLoS ONE 13(6): e0199634, DOI: 10.1371/journal.pone.0199634.

Nechaeva O.B. (2018). Epidemic situation on tuberculosis in Russia // Tuberculosis and lung diseases, 96(8), 15-24.

Shelkova E.S., Romanenko V.V. (2013). Tuberculosis - yesterday, today, tomorrow. Ural Medical Journal, 9,110-120.

The End TB Strategy (2014). WHO, 20.

Tikunov V.S. (1997). Classifications in geography: renaissance or fading? Smolensk: SSU.

Vasilyeva I.A., Belilovsky E.M., Borisov S.E., Sterlikov S.A. (2017). Multidrug-resistant tuberculosis in the countries of the world and the Russian Federation // Tuberculosis and lung diseases, 95(11), 5-17, DOI: 10.21292/2075-1230-2017-95-11-5-17. 